\title{
STUDY OF COMBINED CLEANING PROCESS OF SUNROOT TUBERS
}

\author{
Gregoriy Deynichenko \\ Department of equipment for food and \\ hospitality industry named after M. I. Belyaeva \\ Kharkiv State University of Food Technology and Trade, \\ 333 Klochkivska str., Kharkiv, Ukraine, 61051 \\ DeynichenkoGV@rambler.ru \\ Dmytro Dmytrevskyi \\ Department of equipment for food and \\ hospitality industry named after M. I. Belyaeva \\ Kharkiv State University of Food Technology and Trade \\ 333 Klochkivska str., Kharkiv, Ukraine, 61051 \\ dmitrevskyidv@gmail.com,dmitrevskyi@mail.ru \\ Vitalii Chervonyi \\ Department of equipment for food and \\ hospitality industry named after M. I. Belyaeva \\ Kharkiv State University of Food Technology and Trade \\ 333 Klochkivska str., Kharkiv, Ukraine, 61051 \\ chervonyi.v@gmail.com \\ Oleg Udovenko \\ Department of general engineering disciplines and equipment \\ Donetsk National University of Economics and \\ Trade named after Mykhailo Tugan-Baranovsky \\ 16 Ostrovskyi str., Kryvyi Rih, Ukraine, 50005 \\ Udoleg@mail.ru

\section{Oleksandr Omelchenko} \\ Department of engineering disciplines and equipment \\ Donetsk National University of Economics and \\ Trade named after Mykhailo Tugan-Baranovsky \\ 16 Ostrovskyi str., Kryvyi Rih, Ukraine, 50005 \\ omelchenko84@ukr.net

\section{Olga Melnik} \\ Department of engineering disciplines and equipment \\ Donetsk National University of Economics and \\ Trade named after Mykhailo Tugan-Baranovsky \\ 16 Ostrovskyi str., Kryvyi Rih, Ukraine, 50005 \\ melnikolgaevgenivna@ukr.net
}

\section{Abstract}

Elaboration and improvement of the process of raw material cleaning is an urgent scientifically technical process. The one of most prospective directions of vegetables cleaning process intensification is the elaboration of combined methods of their cleaning. The improvement of tubers cleaning process is based on the combination of thermal processing of sunroot tubers by steam and the process of further mechanical additional cleaning.

The experimental studies of the influence of parameters of the process of sunroot thermal processing by steam on a tuber surface layer transformation were carried out. The influence of the steam pressure and the duration of the process of tubers thermal processing on the depth of the thermal processing of tuber surface layer and also on the effectiveness of the peel separation were 
studied. At the same time there were realized the studies as to the influence of the duration of tubers mechanical additional cleaning process on cleaning quality parameters.

The experimental apparatus and correspondent method that allow to carry out investigations of the combined process of sunroot tubers cleaning with a possibility to determine the influence of all its parameters on the percent of raw material losses and cleaning quality were elaborated. Rational regimes of the combined process of sunroot tubers cleaning were established.

Keywords: sunroot cleaning, combined effect on raw material, cleaning parameters, preliminary thermal processing, mechanical additional cleaning.

\section{Introduction}

The process of vegetables cleaning is a very urgent direction of studies despite the large number of existent methods and equipment for their realization. Cleaning is the one of most laborious procedures at fruit and vegetable raw material processing [1, 2].

At studying cleaning process one must pay attention to such parameters as cleaning quality, waste quantity and also maximal preservation of vitamin and mineral composition of a product [3].

Today the most widespread methods of vegetables cleaning are mechanical and steam ones [4]. But despite the fact that these two methods have many advantages comparing with other ones, shortcomings, typical for each of them, need a detail study of these ways for their more effective use at enterprises of restaurant economy [5]. To shortcomings of the existent equipment may be referred its material and energy intensity, insufficient quality of a product cleaning, big percent of waste, auxillary equipment presence $[6,7]$.

The special attention must be paid to the process of peeling of sunroot tubers of the surface cover. Today this process is very laborious and needs manual labor [8, 9]. At the same time an essential part of a raw material is lost at peeling. It takes place because of a complicated form of sunroot tubers. Under modern production conditions there is a necessity to create the resource-saving equipment, correspondent to the world requirements [10]. Today the one of most prospective directions of sunroot cleaning quality improvement and decrease of raw material losses is a creation of such equipment, which working principle is based on the combined effect of thermal and mechanical processes on a product [11].

Today the absence of complex experimental studies on using the combined effect of these processes on a product essentially complicates the elaboration of new, energetically effective equipment. The solution of the problem of raw material cleaning is the elaboration of combined process of tubers cleaning at the expanse of combination of thermal and mechanical effect on a product. For studying the cleaning process a series of experiment that allow to determine rational parameters of the process realization are necessary.

\section{Materials and Methods}

Taking into account the importance of determination of rational regimes of sunroots tubers cleaning there were realized the studies of the influence of thermal processing parameters and duration of mechanical additional cleaning on the surface layer of sunroot tubers. It was necessary to establish the influence of steam pressure and thermal processing duration on the surface layer of sunroot tubers. The experimental apparatus was designed for studying thermal processing regimes.

The general look of the experimental apparatus is presented on the Fig. 1.

The scheme of the experimental apparatus is presented on the Fig. 2. It consists of the following elements: the frame of apparatus, placed on the stands; the working chamber 11, where the process of sunroot thermal processing by steam of excess pressure takes place, is fixed on it. The steam pressure in the working chamber is determined using the manometer 5 . The working chamber is closed by the cover 9, connected with the flange 10 of the working chamber. Steam from the working chamber is ejected through the outlet valve 6 that is a part of steam-out 7. Steam generator 3 is used for steam generation. The part of the steam generator is filled with water, heated by TEHs. The other part of the steam generator is empty for further filling with steam. At water 
heating at steam generation air must be preliminary ejected from the steam generator. Steam supply in the working chamber from the steam generator is realized through the branch 4 at the open tap 12. For keeping the necessary level of water the steam generator is connected to the central water supply system. At the tap opening water is supplied inside the steam generator through the branch. At safety measures observance the regulation of the water level in the steam generator must be realized only when the pressure in it is equal to the atmospheric one.

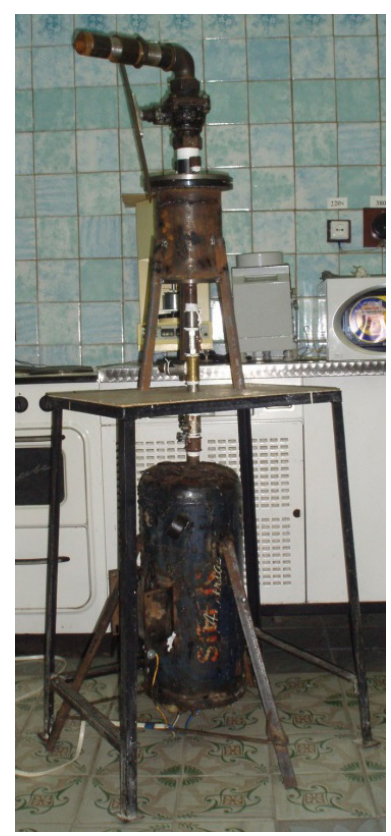

Fig. 1. Experimental apparatus for studying thermal processing influence on the sunroot surface layer

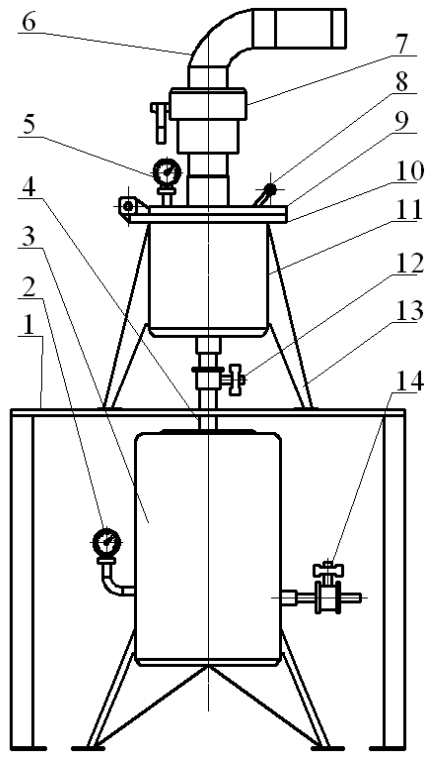

Fig. 2. Scheme of experimental apparatus for studying the thermal processing influence on the sunroot surface level: 1 - frame of experimental apparatus; 2 - manometer of steam generator; 3 - steam generator; 4 - branch of steam supply to the working chamber from the steam generator; 5 - manometer of working chamber; 6 - outlet valve; 7 - steam-out device; 8 - lever for opening the cover of the working chamber; 9 - cover of working chamber;

10 - flange of working chamber; 11 -working chamber; 12 - tap of steam supply to the working chamber; 13 - stands of working chamber; 14 - tap of water supply in the steam generator 
The experimental apparatus for studying the influence of thermal processing on the sunroot surface layer functions in the following way: before starting the work it is necessary to check the water level in the steam generator 3. The proper water level must be set if necessary. After switching on TECs the steam pressure in the steam generator will gradually increase. It must be controlled by the manometer 2 . When the steam generator comes to the working regime, it is necessary to load tubers in the working chamber 11. After that it is necessary to open the tap 12, providing water supply from the steam generator to the working chamber. The duration of the process of sunroot thermal processing by steam was within $10 \ldots 70 \mathrm{~s}$. Thus, the process of sunroot tubers thermal processing by steam of excess pressure takes place. For providing the effect of skin separation from a sunroot tuber it is necessary to realize the instant steam-out from the working chamber. Before that steam supply from the steam generator to the working is stopped. Then it is necessary to open the tap of the steam-out device 7, providing the instant steam-out through the outlet valve 6.

As a result of abrupt steam-out the pressure in the working chamber instantly decreases, due to which, moisture, contained under the tuber peel boils up and transforms into steam that breaks the peel of the product.

On the Fig. 3 are presented the sunroot samples that underwent the preliminary thermal processing by steam and the ones that underwent the further mechanical additional cleaning.

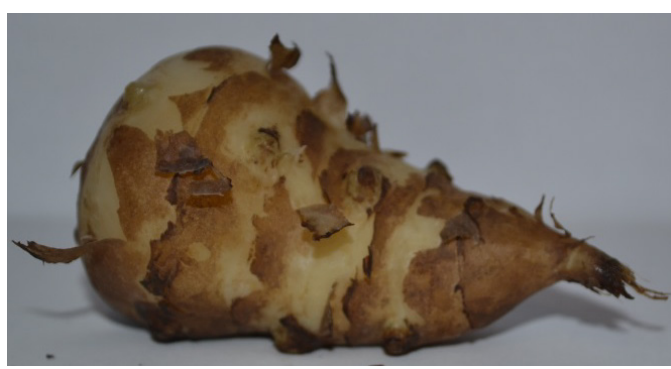

$a$

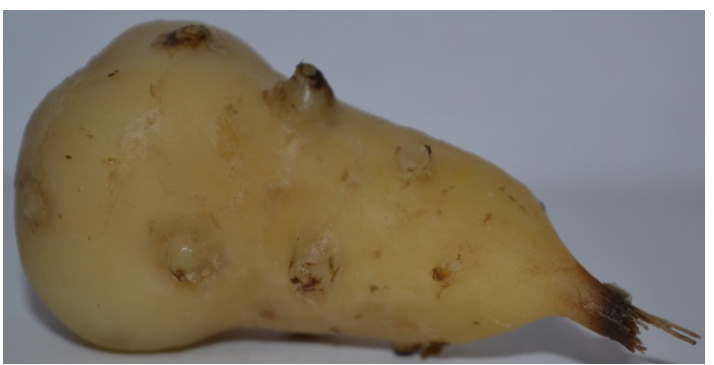

$b$

Fig. 3. Sunroot samples at the combined cleaning process: $a$-after preliminary thermal processing; $b$ - after mechanical additional cleaning

At the process of sunroot thermal processing the steam pressure changes within $0,3 \ldots 0,7 \mathrm{MPa}$ with interval $0,1 \mathrm{MPa}$. The duration of the process of sunroot tubers thermal processing by steam was within $10 . . .70 \mathrm{~s}$. If the duration of the cleaning was less than $10 \mathrm{~s}$, the connection between cells of the sunroot surface layer is destructed for the further process of mechanical additional cleaning. In the case when the thermal processing duration exceeded $70 \mathrm{~s}$, the depth of the sunroot surface layer thermal processing essentially increased that led hereinafter to essential losses of the raw material.

The sunroot processing by steam with the pressure lower than $0,3 \mathrm{MPa}$ also did not provide the necessary separation of skin from a tuber. At the experimental studies on the determination of steam effect on the surface layer, the maximal steam pressure was 0,7 MPa. Such pressure provides a possibility to use the apparatus for tubers cleaning by the combined methods at restaurant economy enterprises.

After studying the process of sunroot tubers thermal processing by steam it is necessary to study the process of their mechanical additional cleaning.

At the offered combined cleaning process abrasive working surfaces are not needed, because the connection between a sunroot peel and a tuber is destructed at the thermal processing by steam. For the process of sunroot mechanical cleaning after steam processing the working surface with wavy form, produced of steel is used. At using it the sunroot skin is peeled by the friction force between tubers and surface. To minimize raw material losses it is necessary to carry out studies for determination of the duration of the mechanical additional cleaning process. This process was carried out for sunroot tubers that underwent the thermal processing.

The percent of cleaned tubers and the one of raw material losses were chosen as quality parameters at the experimental studies of the mechanical additional cleaning process. It becomes 
necessary to establish the dependence of the percent of cleaned tubers and the one of raw material losses on the mechanical additional cleaning duration. The percent of raw material losses was determined by weighing a sunroot before the process of additional mechanical cleaning and after it. It becomes necessary to study the influence of effort value of the sunroot peel separation after the thermal processing on the percent of sunroot tubers cleaning. At the same time it is necessary to determine the depth of the sunroot surface layer thermal processing on the percent of raw material losses. At the studies of the process of sunroot tubers mechanical additional cleaning its storage term was taken into account.

At determination of the percent of cleaned tubers was chosen a sunroot, which effort value of peel separation was within $1,142 \mathrm{~N}-15,0 \mathrm{~N}$. Sunroot tubers with a big effort of peel separation were not chosen because at the process of mechanical additional cleaning their peel was not completely separated from a tube. The duration of the mechanical additional cleaning process changes within $30 \ldots 110 \mathrm{~s}$.

The parameters that influence raw material losses are: the depth of the sunroot tuber surface layer thermal processing, the sunroot storage term and also the duration of the mechanical additional cleaning process. To decrease the percent of sunroot losses at the mechanical additional cleaning it is necessary to minimize the thermal processing depth. But when tubers have significant mechanical and biological lesions, it is necessary to increase the thermal processing depth to eliminate an inedible part of a product. So, tubes which thermal processing depth was $1-5 \mathrm{~mm}$ were selected for the study of the mechanical cleaning process and determination of the percent of raw material losses.

\section{Results}

At the studies there was established the dependence of raw material losses on regimes of the cleaning process realization. The thermal processing depth is $1,0-5 \mathrm{~mm}$. The increase of the steam pressure and the duration of the thermal processing raise the sunroot surface layer thermal processing depth and decrease the effort of peel separation from a tuber. The thermal processing depth is $1,0-5 \mathrm{~mm}$. The effort of peel separation from a sunroot tuber after the thermal processing is within $1,142 \mathrm{~N}-15,0 \mathrm{~N}$.

The increase of the duration of mechanical additional cleaning raises the percent of cleaned sunroot tubers but leads to the growth of raw material losses. It was determined that the decrease of the effort of peel separation from a tuber at the thermal processing allows to decrease the duration of the mechanical additional cleaning process. It was determined that the growth of the thermal processing depth of the sunroot tuber surface layer is attended with the increase of raw material losses.

The steam pressure at the thermal processing must be $0,3 \mathrm{MPa}$ at the duration $35 \ldots 60 \mathrm{~s}$. The duration of the mechanical additional cleaning is within $70 \ldots 105 \mathrm{~s}$. The rational parameters of the mechanical cleaning process for the elaborated apparatus provide the maximally possible rate of cleaning quality - no less than $80 \%$ by the percent of cleaned tubers. At the beginning of the studies on determination of the percent of raw material losses, the general mass of studied sunroot tubers was $5 \mathrm{~kg}$. After the series of experimental studies of the combined cleaning process, the mass of cleaned tubers was $4-4,7 \mathrm{~kg}$.

\section{Conclusions}

The regimes of the combined cleaning process were determined for minimization of raw material losses and improvement of sunroot tubers cleaning quality. There were established the necessary steam pressure and the duration of the preliminary thermal processing and mechanical additional cleaning.

The experimental apparatus for the sunroot tubers cleaning was elaborated. The methodology of the combined cleaning process was also elaborated. The experimental apparatus with this methodology allows to study the sunroot cleaning process taking into account such factors as the thermal processing duration and steam pressure. The percent of cleaned sunroot tubers and the one of raw material losses were determined at the study. The experimental studies of the combined cleaning process allowed to determined its parameters. The rational parameters of the combined 
process of sunroot tubers cleaning allow to minimize raw material losses and to provide the high quality of cleaning of a product. The authors plan to use the elaborated methods for the study of the cleaning process of other tuber types. The presented results of the studies may be used for projecting and improvement of new types of cleaning equipment.

\section{References}

[1] Baselice, A. , Colantuoni, F., Lass, D., Nardone, G., Stasi, A. (2017) Trends in EU consumers' attitude towards fresh-cut fruit and vegetables. Food Quality and Preference, 59, 87-96. doi: 10.1016/j.foodqual.2017.01.008

[2] Poelman, A. A. M., Delahunty, C. M., de Graaf, C. (2017). Vegetables and other core food groups: A comparison of key flavour and texture properties. Food Quality and Preference, 56, 1-7. doi: 10.1016/j.foodqual.2016.09.004

[3] Barbosa, C., Alves, M. R., Rocha, S., Oliveira, M. B. P. P. (2016). Modified atmosphere packaging of precooked vegetables: Effect on physicochemical properties and sensory quality. Food Chemistry, 194, 391-398. doi: 10.1016/j.foodchem.2015.07.147

[4] Khaled, D. El, Castellano, N. N., Gazque, J. A., García Salvador, R. M., Manzano-Agugliaro, F. (2017) Cleaner quality control system using bioimpedance methods: a review for fruits and vegetables. Journal of Cleaner Production, 140, 1749-1762. doi: 10.1016/j.jclepro.2015.10.096

[5] Faour-Klingbeil, D., Todd, E. C. D., Kuri, V. (2016). Microbiological quality of ready-to-eat fresh vegetables and their link to food safety environment and handling practices in restaurants. LWT - Food Science and Technology, 74, 224-233. doi: 10.1016/j.lwt.2016.07.051

[6] Drissner, D., Zuercher, U. (2014). Safety of Food and Beverages: Fruits and Vegetables. Encyclopedia of Food Safety, 253-259. doi: 10.1016/b978-0-12-378612-8.00280-8

[7] Van Herpen, E., Immink, V., van den Puttelaar, J. (2016). Organics unpacked: The influence of packaging on the choice for organic fruits and vegetables. Food Quality and Preference, 53, 90-96. doi: 10.1016/j.foodqual.2016.05.011

[8] Sánchez, C., Baranda, A. B., Martínez de Marañón, I. (2014). The effect of High Pressure and High Temperature processing on carotenoids and chlorophylls content in some vegetables. Food Chemistry, 163, 37-45. doi: 10.1016/j.foodchem.2014.04.041

[9] Ramsay, S. A., Shriver, L. H., Taylor, C. A. (2017). Variety of fruit and vegetables is related to preschoolers' overall diet quality. Preventive Medicine Reports. 5, 112-117. doi: 10.1016/j.pmedr.2016.12.003

[10] Juániz, I., Ludwig, I. A., Huarte, E., Pereira-Caro, G., Moreno-Rojas, J. M., Cid, C., De Peña, M. -P. (2016). Influence of heat treatment on antioxidant capacity and (poly)phenolic compounds of selected vegetables. Food Chemistry, 197, 466-473. doi: 10.1016/j.foodchem.2015.10.139

[11] Fabbri, A. D. T., Crosby, G. A. (2016). A review of the impact of preparation and cooking on the nutritional quality of vegetables and legumes. International Journal of Gastronomy and Food Science, 3, 2-11. doi: 10.1016/j.ijgfs.2015.11.001 